\title{
la consolidation unidimensionnelle sous charge cyclique
}

\section{The one dimensionnal consolidation under cyclic loading}

\author{
I. JURAN \\ Directeur-Adjoint du CERMES* \\ A. BERNARDET \\ Ingénieur Civil des Ponts et Chaussées, \\ Elève-Chercheur au CERMES*
}

\section{Résumé}

Après un rapide exposé des théories existant dans la littérature sur la consolida. tion unidimensionnelle sous charge cyclique, on présente les résultats d'une étude expérimentale sur ce problème, obtenus sur un limon. Deux sortes d'essais ont été réalisés : des essais statiques et cycliques. Une analyse approfondie des résul. tats obtenus avec les premiers a permis d'améliorer considérablement l'interpré. tation des seconds. Cette étude, limitée essentiellement à l'influence de la période des cycles, a cependant mis en évidence l'importance du comportement irréversible des sols fins sur le processus de la consolidation dans le cas d'une charge cyclique.

\begin{abstract}
After a brief review of the published theories of the one dimensionnal consolidation under cyclic loading this paper presents the results of an experimental study of this problem obtained on a silty clay. Two kinds of tests has been performed: static and cyclic tests. A very precise analysis of the results of the first ones had improved considerably the interpretation of the second ones. This study, limited to the effect of the periode of the cycles, had, however, show how important are the effects of the irreversible behavior of fine grained soils on the process of the consolidation in the case of a cyclic load.
\end{abstract}




\section{INTRODUCTION}

Cet article présente une étude expérimentale de la consolidation unidimensionnelle sous charge cyclique. Cette étude a eu essentiellement pour objectif de mettre en évidence l'effet de la période des cycles sur la vitesse de consolidation et sur l'amplitude du tassement.

Pour atteindre cet objectif on a effectué sur un sol limoneux des essais de consolidation œedométrique sous charge cyclique et sous charge statique. Les essais sous charge cyclique ont permis de mettre en évidence un certain nombre de phénomènes et en particulier de montrer que lors d'une consolidation sous un chargement cyclique le sol atteint un état d'équilibre, à pression interstitielle moyenne constante, sans que les surpressions interstitielles provoquées par le chargement appliqué soient entièrement dissipées. En comparant les courbes de consolidation obtenues sous charge cyclique avec celles obtenues sous charge statique on remarque que les cycles ont pour effet de réduire la vitesse de consolidation.

Pour l'interprétation théorique des résultats expérimentaux d'essais sous charge cyclique on a considéré la solution proposée par BALIGH et LEVADOUX (1978) qui s'appuie sur les hypothèses de la théorie de TERZAGHI. D'utilisation courante dans la pratique celle-ci présente l'avantage de conduire à des calculs relativement simples. Mais elle repose sur un certain nombre d'hypothèses simplificatrices, en particulier sur les lois du comportement du sol, entraînant généralement des erreurs significatives dans l'estimation des temps de tassements. Dans le cas d'une charge cyclique, on constate que la solution proposée par BALIGH et LEVADOUX ne permet pas de prévoir correctement les phénomènes observés. Elle permet cependant de les comprendre et d'interpréter ainsi de manière qualitative les résultats expérimentaux.

Dans ce qui suit on rappelle brièvement la solution théorique proposée par BALIGH et LEVADOUX et on présente les résultats expérimentaux, l'analyse des phénomènes observés ainsi qu'une comparaison entre la théorie et l'expérience.

\section{ANALYSE THEORIQUE DE LA CONSOLIDATION UNIDIMENSIONNELLE SOUS CHARGE CYCLIQUE}

\subsection{Réflexions sur l'hypothèse d'un comportement réversible du sol}

La figure 1 montre l'évolution des isochrones des surpressions interstitielles au cours d'une consolidation cedométrique sous un chargement cyclique. Ces isochrones traduisent la variation de la surpression interstitielle $\Delta \mathrm{u}(\mathrm{t})$ avec la profondeur normalisée $\mathrm{Z}=\mathrm{z} / \mathrm{H}$ ( $\mathrm{z}$ et $\mathrm{H}$ étant respectivement la profondeur considérée et le chemin de drainage) à l'instant $t$.

Elles ont été déterminées à partir des formules établies par WILSON et ELGOHARY (1974) qui résultent de l'application de la théorie de TERZAGHI au cas d'un chargement cyclique en prenant pour le sol un comportement (relation indice des vides-contrainte effective) linéaire et réversible. Avant développement elles s'écrivent :

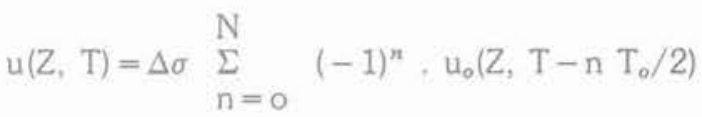

où :

$\mathrm{T}=\mathrm{C}_{y} \cdot \mathrm{t} / \mathrm{H}^{2}$, facteur temps,

$T_{0}$ est la période exprimée en facteur temps $\frac{\mathrm{C}_{v} t_{o}}{\mathrm{H}^{2}}$

$\mathrm{C}_{v}$ est le coefficient de consolidation

$\Delta \sigma$ est l'amplitude de la charge cyclique

to est la période des cycles

$\mathrm{N}$ est le nombre des cycles

où : $u_{\circ}(Z, T)$ note la solution de base de léquation de TERZAGHI pour une variation unitaire de la charge appliquée.

L'analyse de l'évolution de ces isochrones au cours des premiers cycles fait apparaître que pendant la première phase de chargement la pression interstitielle $\Delta u(t)$ se dissipe de la même façon que lors d'une consolidation sous une charge statique égale à $\Delta \sigma$ et atteint l'isochrone 1 (fig. 1) au moment du premier déchargement. Ce déchargement s'accompagne instantanément d'une diminution uniforme égale à $\Delta \sigma$ de la pression interstitielle qui devient négative pour la totalité de la couche de sol (isochrone 2). Si l'on compare l'isochrone 3 , atteinte au cours de la phase de déchargement, à l'isochrone 2 , on peut remarquer que dans la partie supérieure de la couche, proche du drainage, la pression interstitielle a crû. La contrainte effective a donc diminué. Au contraire, dans la partie inférieure, la pression interstitielle a continué à décroître et la contrainte effective à augmenter.

On constate donc qu'à un même instant une partie de la couche de sol est à l'état surconsolidé alors que l'autre reste à l'état normalement consolidé. Or, en pratique, les seuls sols susceptibles de présenter un comportement réversible sont les sols surconsolidés. En effet, la plupart des sols présentent une compressibilité différente suivant que la contrainte effective est égale ou inférieure à la contrainte de préconsolidation $\left(\sigma_{p}\right)$.

\subsection{Caractérisation d'un comportement irréversible}

Tout en s'appuyant sur les hypothèses de la thérorie de TERZAGHI, BALIGH et LEVADOUX (1978) ont pris en compte l'effet de la contrainte de préconsolidation sur la relation indice des vides-contrainte effective. Celleci est schématisée par deux droites (fig. 2) l'une de pente $\mathrm{a}_{v}$ lorsque le sol est normalement consolidé $\left(\sigma^{\prime}=\sigma_{p}^{\prime}\right)$ l'autre de pente $a_{\text {ysc }}$ lorsque le sol est surconsolidé $\left(\sigma^{\prime}<\sigma_{p}^{\prime}\right)$. Pour chacun de ces deux états les coefficients de consolidation sont respectivement $C_{y}$ et $C_{v s c}$.

Ils ont caractérisé un tel comportement en introduisant les deux paramètres suivants :

$$
\begin{array}{cc}
\alpha=\frac{\mathrm{a}_{v s c}}{\mathrm{a}_{v}} & \beta=\frac{\mathrm{C}_{v}}{\mathrm{C}_{v s c}} \\
0<\alpha<1 & 0<\beta<1
\end{array}
$$




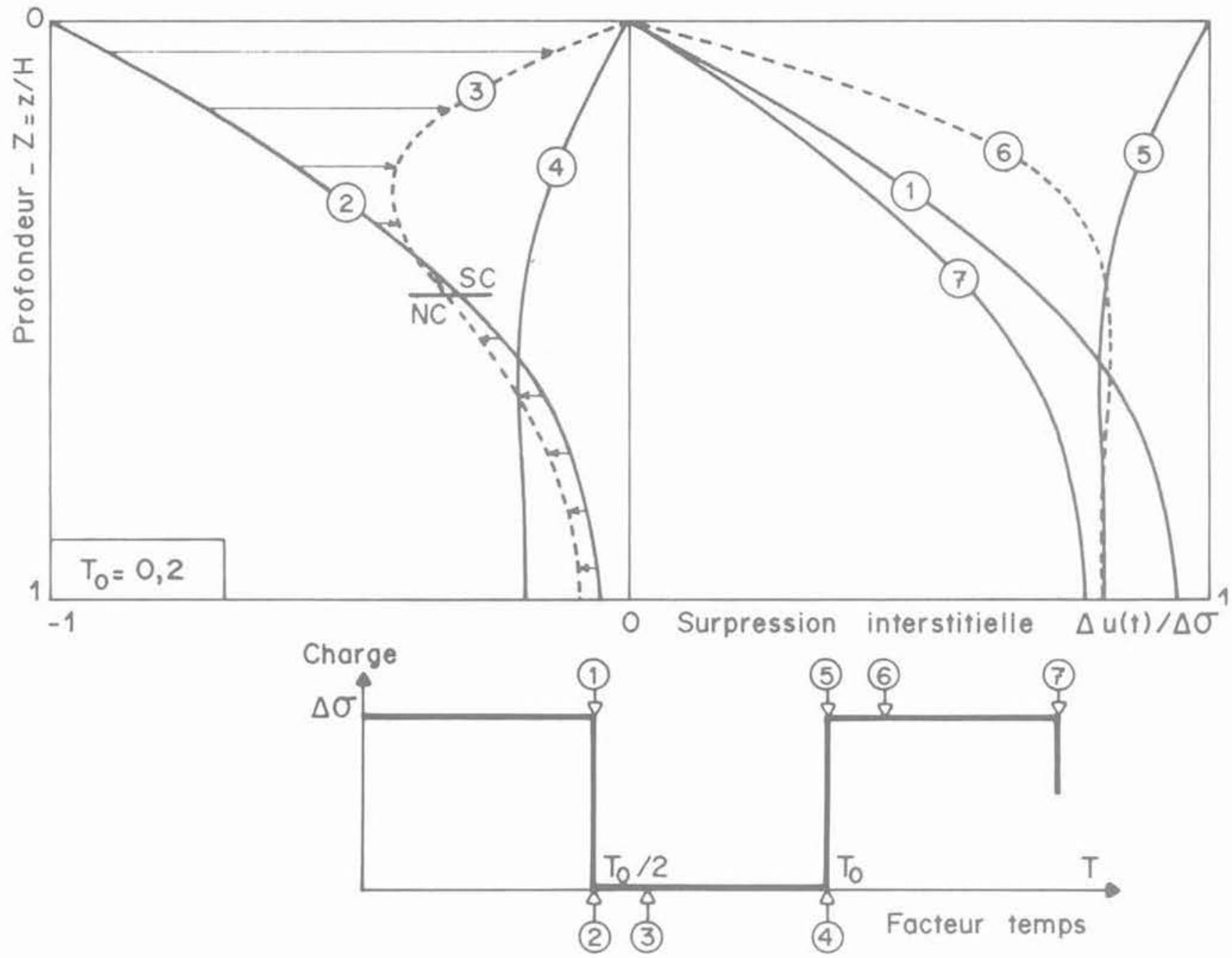

Fig. 1 - Evolution des isochrones au cours des premiers cycles d'après la théorie de TERZAGHI.

qu'ils supposent constants au cours de la consolidation et indépendants de la contrainte de préconsolidation $\sigma_{p}^{\prime}$. Lorsque $\alpha=\beta=1$, on se retrouve dans le cas d'un sol à comportement réversible.

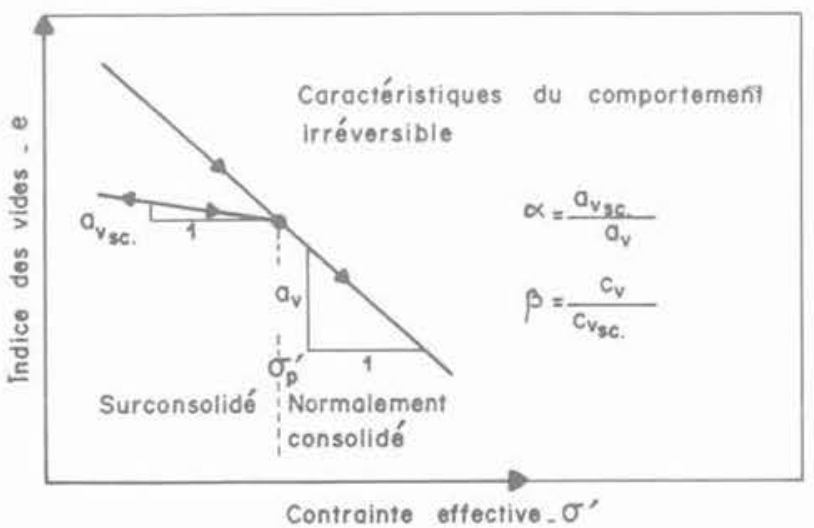

Fig. 2 - Schématisation d'un comportement irréversible (BALIGH et LEVADOUX, 1978).

\subsection{Solutions à l'état d'équilibre}

Lorsque le nombre de cycles devient suffisamment grand, le sol atteint un état d'équilibre à pression interstitielle moyenne constante et les tassements arrivent alors à leur amplitude maximum. A l'état d'équilibre, sous charge cyclique, le sol n'est donc pas entièrement consolidé et en maintenant à l'arrêt des cycles la charge maximale appliquée constante on provoque une reprise du tassement de consolidation primaire. Celle-ci est due à la dissipation de la surpression interstitielle qui réside dans le sol à l'état d'équilibre sous la charge cyclique. Par définition de l'état d'équilibre sous charge cyclique, la contrainte de préconsolidation ne varie pas d'un cycle à l'autre. Par conséquent, les caractéristiques de consolidation sont en tous points et à chaque instant celles du sol surconsolidé. BALIGH et LEVADOUX (1978) ont montré qu'alors les répartitions des pressions interstitielles, contraintes effectives et contraintes de préconsolidation, sont celles obtenues en considérant un sol à comportement réversible avec un coefficient de consolidation $\mathrm{C}_{v s c}=\mathrm{Cv} / \beta$. Elles sont parfaitement définies et peuvent être déterminées à partir de résultats de la 
théorie de TERZAGHI. Ainsi, l'état d'équilibre ne dépend que de la période des cycles exprimées en facteur temps pour le sol surconsolidé $T_{0} / \beta$

(où : $\mathrm{T}_{0}=\mathrm{C}_{0} \mathrm{t}_{0} / \mathrm{H}^{2} ; \mathrm{t}_{\mathrm{o}}$ étant la période des cycles).

La connaissance simultanée des répartitions de la contrainte effective $\sigma^{\prime}(z)$ et de la contrainte de préconsolidation $\sigma_{p}^{\prime}(\mathrm{z})$ à un instant donné suffit pour déterminer le tassement. Compte tenu de la loi de comportement bilinéaire retenue (fig. 2), l'indice des vides e est donné par :

$$
e-e_{0}=-\lambda_{v} \Delta \sigma_{p}^{\prime}(\mathrm{Z})+\alpha \mathrm{a}_{\nu}\left[\Delta \sigma_{p}^{\prime}(\mathrm{Z})-\Delta \sigma^{\prime}(\mathrm{Z})\right]
$$

où $e_{0}$ est l'indice des vides initial.

On en déduit le degré de consolidation U défini par :

$\mathrm{U}=\frac{\Delta \mathrm{h}(\mathrm{t})}{\Delta \mathrm{h}_{f}}=\int_{0}^{1} \frac{\Delta \sigma^{\prime}(\mathrm{Z})}{\Delta \sigma} \mathrm{dZ}+(1-\alpha) \int_{0}^{1} \frac{\left[\Delta \sigma_{p}^{\prime}(\mathrm{Z})-\Delta \sigma^{\prime}(\mathrm{Z})\right]}{\Delta \sigma} \mathrm{dz}$

où : $\Delta h(t)$ note le tassement à l'instant $\mathrm{t}$;

$\Delta \mathrm{h}_{f}$ note le tassement final sous la charge statique $\Delta \sigma$.

L'irréversibilité du comportement du sol est exprimée par la seconde intégrale qui disparaît lorsque $\alpha=1$.

On remarque qu'à l'état d'équilibre le degré de consolidation oscille à l'intérieur d'un fuseau défini par deux valeurs extrêmes correspondant respectivement à un tassement maximal $U^{\infty}{ }_{\max }$ et un tassement minimal $\mathrm{U}^{\infty}{ }_{\text {min }}$. Pour déterminer ces deux valeurs, on considère que :

$$
\Delta \sigma_{\max }^{\prime}(\mathrm{Z})+\Delta \sigma_{\min }^{\prime}(\mathrm{Z})=\Delta \sigma
$$

où : $\Delta \sigma_{\max }^{\prime}$ note la contrainte effective à la fin d'une phase de chargement et $\Delta \sigma^{\prime}{ }_{\min }$ à la fin d'une phase de déchargement.

En supposant de plus que :

$$
\Delta \sigma_{\max }(\mathrm{Z})=\Delta \sigma_{p}^{\prime}
$$

On obtient :

$$
\begin{aligned}
& \text { a. } U^{\infty}{ }_{\max }=\int_{0}^{1} \frac{\Delta \sigma_{\max }(\mathrm{Z})}{\Delta \sigma} \mathrm{dZ}=1-2 \underbrace{\infty}_{\mathrm{m}=0} \\
& \frac{e^{-M^{2}} T_{0} / 2 \beta}{M^{2}\left(e^{\left.-M^{2} T_{0} / 2 \beta+1\right)}\right)} \\
& \text { b. } U^{\infty}{ }_{\min } \alpha+(1-2 \alpha) U^{\infty}{ }_{\max } \\
& \text { où : } M=(2 m+1) \pi / 2
\end{aligned}
$$

\subsection{Evolution du tassement avec le temps}

La bilinéarité de la loi de comportement prise en compte conduit à une hétérogénisation de la couche de sol au cours de consolidation qui ne permet pas de résoudre analytiquement le problème. La solution exacte ne peut être obtenue que par des méthodes de résolution numériques. Cependant, certaines hypothèses sur la dissipation des pressions interstitielles au cours des cycles ont permis à BALIGH et LEVADOUX (1978) de détermi- ner simplement un encadrement des courbes enveloppes du tassement réel $\mathrm{U}_{\max }$ et $\mathrm{U}_{\text {min }}$.

Les auteurs ont supposé qu'au début de chaque phase de chargement :

- les pressions interstitielles ont une répartition initiale uniforme et sont telles que : $\Delta \mathrm{u}=\Delta \sigma$,

- la contrainte de préconsolidation est la contrainte effective atteinte à la fin de la phase de chargement précédente.

Il en découle que les isochrones sont données en permanence par la solution de base de TERZAGHI pour une charge statique : u $(Z ; T)$. On montre en particulier pour le cas d'un chargement cyclique qu'il existe un facteur temps $\mathrm{T}_{N}$ tel que l'isochrone atteinte, à la fin de chaque phase de chargement, est donnée par :

$\mathrm{u}\left(\mathrm{Z} ; \mathrm{NT}_{o} / 2\right)=\Delta \sigma \cdot \mathrm{u}_{0}\left(\mathrm{Z}, \mathrm{T}_{N}\right)$ avec $\mathrm{N}$ impair BALIGH et LEVADOUX (1978) ont montré que le facteur temps $\mathrm{T}_{N}$ est donné par :

$$
\mathrm{T}_{N}=\frac{\mathrm{T}_{0}}{2 \beta}\left[1-(1-\beta)^{\frac{N+1}{2}}\right]
$$

L'analyse des isochrones au cours d'un cycle montre que d'une part, pendant la phase de décharge, la dissipation du gradient des supressions interstitielles provoque initialement une augmentation de la contrainte effective dans la partie basse de l'échantillon et par conséquent la contrainte de préconsolidation $\sigma_{p}^{\prime}$ y est supérieure à la contrainte effective $\sigma_{\max }^{\prime}$ atteinte à la fin de la phase de chargement. Par ailleurs, à la fin d'une phase de déchargement les pressions interstitielles négatives ne sont pas nulles. Les hypothèses considérées par BALIGH et LEVADOUX conduisent donc à sousestimer les contraintes effectives et par voie de conséquence, le tassement. Ils obtiennent ainsi une limite inférieure $\mathrm{U}_{\max }^{\mathrm{li}}\left(\mathrm{NT}_{\mathrm{o}} / 2\right)$ du tassement maximal en fin d'une phase de chargement.

Pour obtenir un encadrement du tassement réel ils déterminent une borne supérieure $\mathrm{U}^{\mathrm{ls}}{ }_{\max }$ en considérant un majorant de la différence

$$
\left.\Delta \mathrm{U}=\mathrm{U}_{\max }\left(\mathrm{NT}_{\mathrm{o}} / 2\right) \cdot \mathrm{U}_{\max }^{\mathrm{li}} \mathrm{NT}_{\mathrm{o}} / 2\right)
$$

Ce terme croissant avec le nombre de cycles tend donc vers un maximum lorsque $\mathrm{N}$ tend vers l'infini. Cette valeur limite est donnée par :

$$
\begin{gathered}
\Delta \mathrm{U}_{\max }=\mathrm{U}_{\text {max }}^{\infty}-\mathrm{U}_{0}\left(\mathrm{~T}_{0} / 2 \beta\right) \\
\text { et donc : } \mathrm{U}^{\text {ls }}{ }_{\max } \mathrm{U}_{\text {max }}^{\mathrm{ll}}\left(\mathrm{NT}_{0} / 2\right)+\Delta \mathrm{U}_{\max }
\end{gathered}
$$

Au cours de la consolidation sous la charge cyclique le tassement réel passe progressivement de la limite inférieure, pour les premières valeurs de $\mathrm{N}(1,3 \ldots)$, à la limite supérieure atteinte lorsque $\mathrm{N}$ tend vers l'infini. Or l'écart entre ces deux limites $\Delta \mathrm{U}_{\max }$ est fonction de la période des cycles $T_{0} / \beta$ (fig. 3 ) et tant que celle-ci est suffisamment longue ( $>1$ par exemple) il reste dans des limites raisonnables $(<5,3 \%)$. Par contre, lorsque la période devient petite, les deux limites s'écartent l'une et l'autre et, pour évaluer la vitesse de consolidation, il serait nécessaire d'avoir un encadrement plus pré. cis de la courbe de tassement réelle ce qui demande de faire appel à une méthode de résolution numérique. 


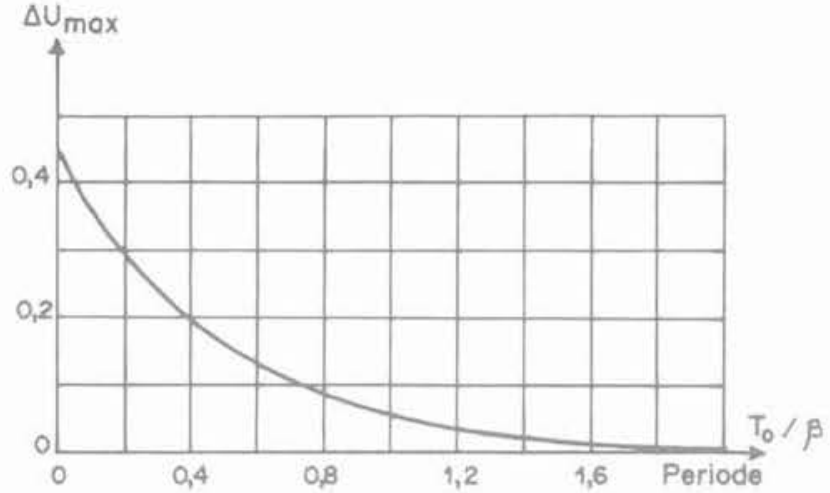

Fig. 3 - Variations avec la période de l'écart $\Delta U_{\max }$ entre les deux bornes d'encadrement de $U_{\max }$

La courbe enveloppe des tassements minimaux $\mathrm{U}_{\min }$ étant de moindre importance dans la pratique les auteurs n'en ont déterminé qu'une borne inférieure obtenue à partir de la courbe des tassements maximaux. Pour ce faire, ils ont repris tout d'abord l'hypothèse faite pour évaluer $\mathrm{U}_{\text {max }}^{\mathrm{li}}\left(\mathrm{NT}_{o} / 2\right)$, à savoir que la pression interstitielle revient à zéro au cours d'une phase de déchargement. On obtient ainsi une première valeur pour cette borne inférieure par:

$\mathrm{U}_{\min }\left(\mathrm{NT}_{o} / 2\right)=(1-\alpha) \mathrm{U}_{\max }\left(\mathrm{N}-1 \mathrm{~T}_{0} / 2\right) ; \mathrm{N}$ pair

On en obtient une seconde en supposant que le regonflement $\left[\mathrm{U}_{\max }-\mathrm{U}_{\min }\right]$ est le même au cours de chaque cycle. Or ce terme atteint sa valeur maximale à l'état d'équilibre et donc :

$\mathrm{U}_{\min }\left(N \mathrm{NT}_{\alpha} / 2\right)=\mathrm{U}_{\max }\left[(\mathrm{N}-1) \mathrm{T}_{\rho} / 2\right]-\alpha\left(2 \mathrm{U}_{\max }^{\infty}-1\right)$

On ne retiendra pour $\mathrm{U}_{\min }$ que la plus élevée de ces deux valeurs. Pour les premières valeurs de $\mathrm{N}$, celle-ci est donnée par la relation (8), puis par (9) lorsque $\mathrm{N}$ devient suffisamment grand.

\subsection{Exemple de courbe de consolidation}

La figure 4 présente une courbe de consolidation sous un chargement cyclique obtenue en considérant la solution décrite ci-dessus avec une période de cycles de $\mathrm{T}_{0}=0.1$ et $\alpha=\beta=0.1$.

Sur cette figure on distingue pour chaque cycle à la fin de la phase de chargement les deux limites supérieure et inférieure entre lesquelles passe la courbe enveloppe des tassements maximaux. En comparant cette solution avec celle obtenue pour le cas d'un sol à comportement réversible, on peut en particulier remarquer que :

- Dans le cas d'un sol à comportement réversible les courbes enveloppes $\mathrm{U}^{\infty}{ }_{\max }$ et $\mathrm{U}^{\infty}{ }_{\min }$ sont à peu près symétriques par rapport à la courbe correspondant à la moitié de la courbe de consolidation sous charge statique $\left(\mathrm{U}_{\mathrm{s}} / 2\right)$. A l'état d'équilibre, on a en particulier la relation $U_{\max }^{\infty}+U_{\min }^{\infty}=1$.

- Dans le cas d'un sol à comportement irréversible les courbes enveloppes $\mathrm{U}_{\max }$ et $\mathrm{U}_{\min }$ ne sont plus symétriques par rapport à la courbe de $U_{s} / 2$ et pour une même période des cycles les tassements extrêmes à l'état d'équilibre sont plus élevés que ceux obtenus pour le sol à comportement réversible.

- Les regonflements au cours des phases de déchargement sont nettement plus faibles que ceux obtenus pour un sol à comportement réversible.

- Les temps de consolidation sont plus élevés. En effet. pour un facteur temps de 2 , contrairement à ce que l'on observe sous une charge statique les tassements extrêmes ne sont pas encore atteints. Ainsi, les cycles ont pour effet de réduire la vitesse de consolidation.

\subsection{Critique de la solution proposée par BALIGH et LEVADOUX}

En plus du fait que la solution proposée par BALIGH et LEVADOUX est inadaptée à l'étude des cycles de courte période, les principales critiques que l'on peut formuler à son encontre sont celles de la théorie de TERZAGHI. Outre le fait que cette théorie ne tient pas compte de l'effet de la consolidation secondaire, ces cri. tiques concernent essentiellement :

- l'hypothèse sur la loi de comportement du sol, en effet, le sol n'est pas linéairement élastique et son coefficient de compressibilité $a_{v}$ varie au cours de la consolidation en fonction de la contrainte effective :

- lapplication de la loi de DARCY supposant une perméabilité constante du sol. En effet, la.perméabilité du sol est fonction de son indice des vides et varie donc avec la contrainte effective au cours de la consolidation.

Par suite des variations de la compressibilité et de la perméabilité du sol au cours de l'essai, le sol n'est pas homogène et la vitesse de consolidation est généralement différente de celle prévue par la théorie de TERZAGHI.

L'utilisation de la solution décrite ci-dessus pour une analyse de la consolidation sous charge cyclique doit donc conduire à sous-estimer le degré de consolidation réel du sol. Cependant, comme on le montre ci-dessous. elle permet une interprétation qualitative des phénomènes observés expérimentalement.

\section{ETUDE EXPERIMENTALE}

\subsection{Objectif et méthodologie}

Cette étude a pour objectif de mettre en évidence l'effet des cycles sur la vitesse de consolidation et sur l'amplitude des tassements. Pour évaluer cet effet des cycles on a effectué deux séries d'essais de consolidation unidimensionnelle à l'cedomètre, à savoir :

- essais de consolidation sous charge statique

- essais de consolidation sous charge cyclique.

Les essais sous charge statique ont été effectués suivant la procédure classique de l'essai oedométrique. Il s'agit d'un chargement par paliers de contrainte successifs maintenus chacun pendant 24 heures avec un doublement de la contrainte à chaque palier.

Pour chaque palier on obtient ainsi une courbe de consolidation correspondant à la variation du degré de con- 
solidation $\mathrm{U}=\Delta \mathrm{h}(\mathrm{t}) / \Delta \mathrm{h}_{s}$ (où : $\Delta \mathrm{h}(\mathrm{t})$ et $\Delta \mathrm{h}_{s}$ sont respectivement le tassement à linstant t et le tassement final) en fonction du temps. La valeur du coefficient de consolidation $\mathrm{C}_{v}$ pour le palier de contrainte considéré est déterminé par la méthode classique de Casagrande. Ainsi, les essais statiques ont permis de déterminer les caractéristiques du sol (compressibilité $a_{v}$ et perméabilité k) à l'état normalement consolidé. Par ailleurs, des essais œdométriques spécifiques, avec des paliers de déchargement, ont été effectués pour déterminer les caractéristiques du sol (compressibilité a $a_{\text {usc }}$ et perméabilité $k_{s c}$ ) à l'état surconsolidé. Ces essais ont permis une détermination expérimentale des paramètres $\alpha$ et $\beta$, définis par BALIGH et LEVADOUX (fig. 2) dont les valeurs ont été utilisées pour l'interprétation des essais sous charge cyclique.

Les essais sous charge cyclique ont été effectués lors du palier correspondant à une contrainte de préconsolidation de $\sigma_{p}^{\prime}=50 \mathrm{kPa}$ avec des cycles ayant une amplitude de $\Delta \sigma=50 \mathrm{kPa}$ et une période $\mathrm{t}_{\mathrm{o}}$ variable suivant les échantillons. Lors des paliers précédents et suivants on a effectué des essais de consolidation sous charge statique. Lors des essais sous une charge cyclique le nombre de cycles a été généralement suffisant pour atteindre un état d'équilibre, à surpression interstitielle moyenne constante, correspondant à la fin de la consolidation primaire sous la charge cyclique appliquée. A l'arrêt des cycles on a maintenu la charge maximale appliquée (100 kPa) constante. On a alors remarqué une reprise du tassement de consolidation primaire due à une dissipation de la surpression interstitielle qui réside dans l'échantillon à l'état d'équilibre sous la charge cyclique. La courbe de consolidation sous un chargement cyclique se présente sous la forme de la variation d'un degré de consolidation $U$ en fonction du facteur temps $T$ défini pour le sol à l'état normalement consolidé $\left(\mathrm{T}=\mathrm{C}_{v} \mathrm{t} / \mathrm{H}^{2}\right)$. Le degré de consolidation est donné par :

$$
\mathrm{U}=\frac{\Delta \mathrm{h}(\mathrm{t})}{\Delta \mathrm{h}_{\mathrm{c}}}
$$

où : $\Delta h(t)$ est le tassement à l'instant $t$;

et : $\Delta \mathrm{h}_{c}$ est le tassement final atteint à la fin de la consolidation primaire du sol sous la charge maximale maintenue constante après l'arrêt des cycles. Pour déterminer ce tassement on utilise la procédure classique de Casagrande d'une façon identique à celle utilisée pour une courbe de consolidation sous une charge statique.

Afin d'évaluer l'effet des cycles sur la vitesse de consolidation et sur lamplitude des tassements on a comparé les courbes de consolidation sous charge cyclique avec une courbe de référence correspondant à la consolidation statique.

La méthode la plus simple pour effectuer une telle comparaison consiste à comparer une courbe de consolidation sous charge cyclique avec une courbe sous charge statique obtenues sur deux échantillons distincts pour le même palier de contrainte, mais compte tenu des écarts observés entre les échantillons cette méthode n'est pas la plus précise. L'analyse statistique de l'ensemble des essais effectués sous charge statique a montré qu'il convient d'estimer pour un palier donné les valeurs du tassement total $\Delta \mathrm{h}_{s}$ et du $\mathrm{t}_{50}$ (temps nécessaire pour atteindre un degré de consolidation de $50 \%$ ) à partir des résultats obtenus sur le même échantillon pour les paliers précédents et suivants. Connaissant les valeurs de $\Delta \mathrm{h}_{s}$ et du $\mathrm{t}_{50}$ on peut établir la courbe de référence correspondant à la consolidation statique pour le palier considéré. Cette procédure a permis d'améliorer de façon significative la précision et l'évaluation de l'effet des cycles sur la vitesse de consolidation.

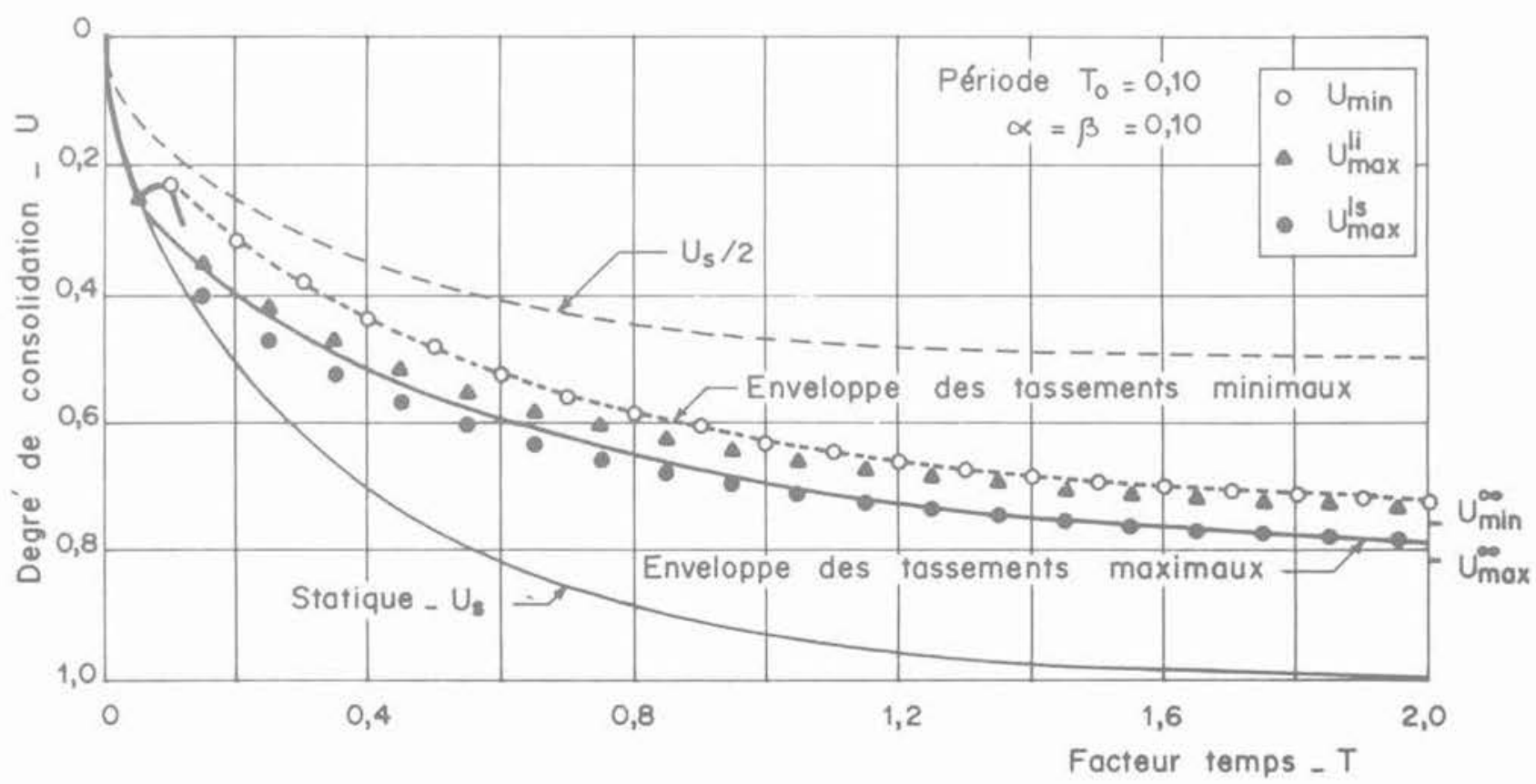

Fig. 4 - Exemple de courbe de consolidation sous charge cyclique. Cas d'un sol à comportement irréversible. BALIGH et LEVADOUX (1978). 
On décrit ci-après les résultats expérimentaux des essais effectués sous charge statique et sous charge cyclique ainsi que l'interprétation de ces derniers fondée sur la solution proposée par BALIGH et LEVADOUX.

\subsection{Matériel et procédure d'essais}

Les essais de consolidation unidimensionnelle ont été effectués pour la plupart à l'œedomètre classique. Cependant, afin d'étudier l'effet des cycles sur la dissipation des surpressions interstitielles on a utilisé également un œedomètre à contre-pression conçu par ROWWE (1966) dont une coupe schématique est représentée à la figure 5.

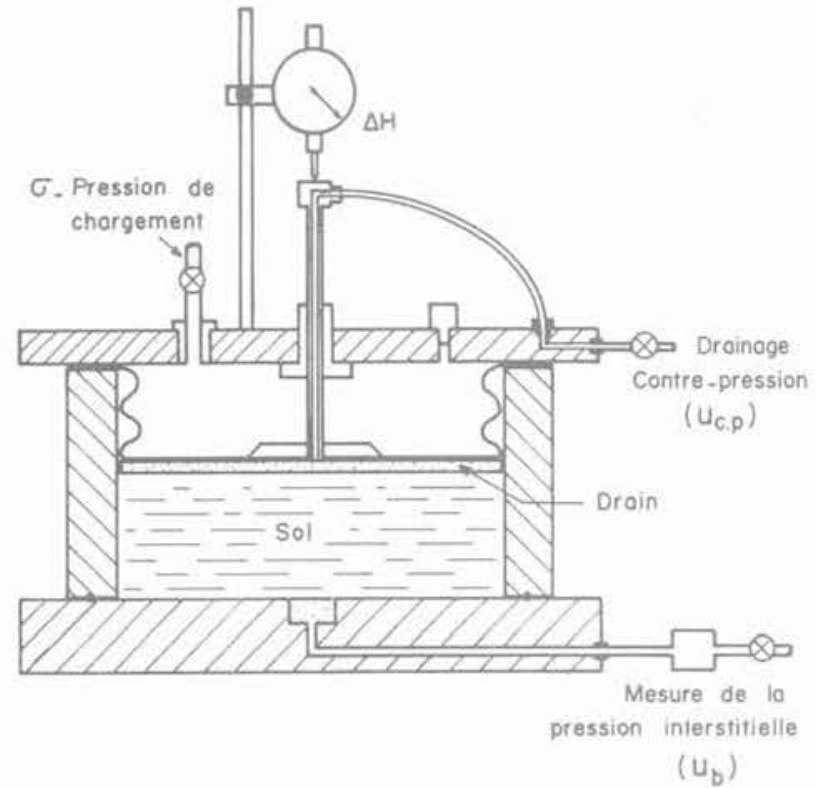

Fig. 5 - Cellule de Rowe : schéma de principe (Rowe, 1966).

Cette cellule permet un chargement hydraulique sur des échantillons cylindriques de $76 \mathrm{~mm}$ de diamètre et de $35 \mathrm{~mm}$ de hauteur initiale ainsi que l'application d'une contrepression permettant d'assurer la saturation du sol. La pression interstitielle est mesurée à la base de l'échantillon par l'intermédiaire d'une pierre poreuse reliée à un capteur de pression électronique.

Sur la cellule de Rowe le chargement cyclique a été obtenu au moyen d'un système de deux électrovannes interposées sur le circuit de mise en pression et commandées par un montage électronique produisant un signal cyclique carré. Ce système a permis d'automatiser l'exécution du chargement cyclique et d'éviter ainsi toute manipulation en cours d'essai.

Il est à noter que la mesure des pressions interstitielles sur la cellule de Rowe s'avère délicate ; la parfaite saturation du sol et des circuits de l'appareil n'est pas toujours aisée à obtenir et cela entraîne un temps de réponse non négligeable sur les indications données par le capteur de pression. En particulier, ce capteur n'est pas en mesure de rendre fidèlement compte des discontinuités de pressions interstitielles provoquées par une variation instantanée de la charge appliquée. Pour contourner ces problèmes de mesure les cycles ont été exécutés en agissant sur la contre-pression $\mathrm{u}_{\mathrm{cp}}$ et en maintenant la charge totale $\sigma$ constante. On note qu'une variation instantanée de la contrepression a un effet tout à fait analogue à celui produit par une variation instantanée de la charge lors d'un chargement ou d'un déchargement. Il en résulte une variation instantanée de la surpression interstitielle qui est égale, au signe près, à celle de la contrepression. Cependant, en procédant ainsi on évite toute variation brusque de la pression interstitielle à la base de l'échantillon. Celle-ci ne varie qu'au cours de la consolidation, lors de la dissipation de la surpression interstitielle; sa variation est donc continue et égale, au signe près, à celle de la contrainte effective à la base de Péchantillon.

\subsection{Caractéristiques du sol et préparation des échantillons}

Le sol choisi pour réaliser cette étude est le limon de Jossigny. Sa courbe granulométrique est donnée sur la figure 6 et ses limites d'Atterberg sont : $w_{L}=35$, $\mathrm{w}_{p}=21$ et $\mathrm{I}_{p}=14$. A titre indicatif les valeurs des principales caractéristiques de consolidation du sol à l'état remanié sont :

$$
\begin{aligned}
& \mathrm{C}_{v} \text { de } 10^{-4} \text { à } 10^{-3} \mathrm{~cm}^{2} / \mathrm{s} \\
& \mathrm{k}_{v} \text { de } 10^{-8} \text { à } 10^{-7} \mathrm{~cm} / \mathrm{s}
\end{aligned}
$$

$\mathrm{C}_{c}$ indice de compressibilité, voisin de 0,25

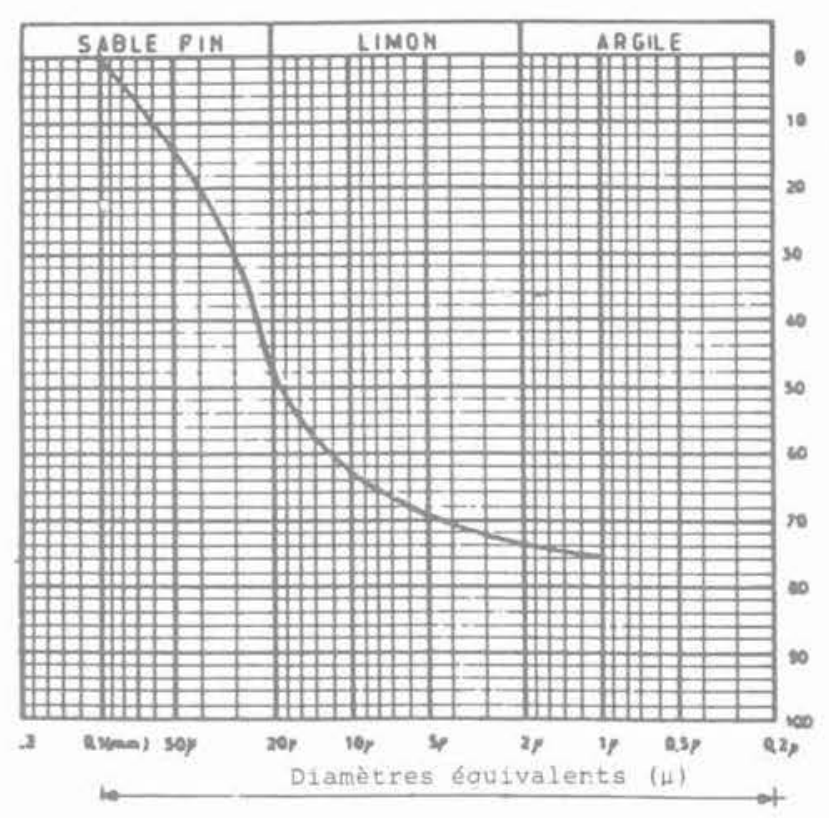

Fig. 6 - Granulométrie du limon.

La qualité des résultats expérimentaux dépend pour une grande part du mode de préparation des échantillons. Pour obtenir des échantillons normalement consolidés et des résultats reproductibles, le sol a été remanié et, après un tamisage humide au tamis de $80 \mu \mathrm{m}$, préparé par malaxage à une teneur en eau de $50 \%$ correspondant à 1,4 fois sa limite de liquidité. 


\subsection{Résultats expérimentaux d'essais de consolidation sous charge statique}

La figure 7 présente des exemples de courbes de consolidation sous charge statique obtenues à l'œdomètre classique d'une part et à la cellule de Rowe d'autre part.

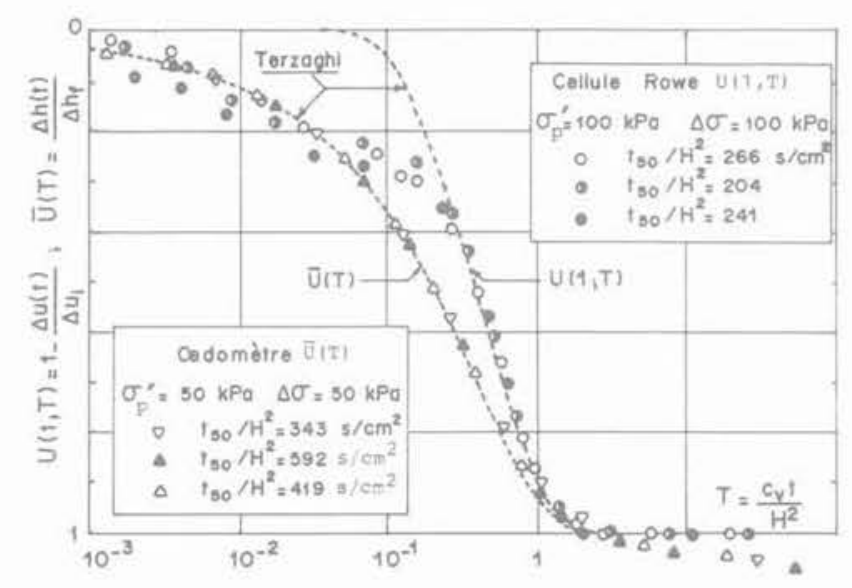

Fig. 7 - Consolidation sous charges statiques à l'œdomètre et à la cellule Rowe.

Comparaison avec la théorie de TERZAGHI.

- Les courbes obtenues à l'œedomètre classique donnent les variations, en fonction du facteur temps. $T$, du degré de consolidation $\bar{U}(\mathrm{~T})$ défini par:

$$
\overline{\mathrm{U}}(\mathrm{T})=\frac{\Delta \mathrm{h}(\mathrm{T})}{\Delta \mathrm{h}_{f}}
$$

où : $\Delta h(T)$ note le tassement à l'instant $\mathrm{T}$;

$\Delta \mathrm{h}_{f}$ note le tassement final.

- Les courbes obtenues à la cellule de Rowe donnent les variations du degré de consolidation U(1, T) à la base de l'échantillon. Celui-ci est déterminé à partir des mesures de la surpression interstitielle à la base de l'éprouvette à la cellule de Rowe par :

$$
\mathrm{U}(1, \mathrm{~T})=1-\frac{\Delta \mathrm{ub}(\mathrm{t})}{\Delta \mathrm{u}_{i}}
$$

où : $\mathrm{u}_{b}(\mathrm{t})$ et $\Delta \mathrm{u}_{i}$ sont respectivement la surpression interstitielle mesurée à l'instant t et celle mesurée initialement, $\mathrm{T}$ note un facteur temps défini en considérant la valeur du $t_{50}$ relevée sur la courbe de tassement correspondante, soit :

$$
T=T(50 \%) \cdot t / t_{50}
$$

$T(50 \%)$ est le facteur temps correspondant à un degré de consolidation $U=50 \%$.

Il est à noter que toutes les courbes de consolidation établies à partir des mesures de tassement sont de forme très proche de celle de la courbe théorique de Terzaghi. Cependant, ces résultats montrent une forte dispersion entre les valeurs de $t_{50} / \mathrm{H}^{2}$ obtenues pour le même palier de charge. Les courbes de dissipation des surpressions interstitielles font apparaître pour des degrés de consolidation inférieurs à $35 \%$ un certain écart entre la courbe de référence déduite de la théorie de TERZAGHI et l'expérience. Cet écart est probablement dû à l'effet d'une légère surconsolidation lors de la mise en place de l'échantillon. Cependant, pour les degrés de consolidation supérieurs à $35 \%$, on observe un très bon accord entre les résultats expérimentaux et la théorie de TERZAGHI. Il en résulte que la valeur du coefficient de consolidation $\mathrm{C}_{v}$ déterminée à partir des mesures de pressions interstitielles est pratiquement identique à celle déterminée à partir des mesures de tassements.

La figure 8 présente les variations du rapport $t_{50} / \mathrm{H}^{2}$, caractérisant la vitesse de consolidation, en fonction de la contrainte effective. Les valeurs de $t_{50} / \mathrm{H}^{2}$ sont des valeurs moyennes obtenues en considérant l'ensemble des essais effectués respectivement à l'œedomètre classique et à la cellule de Rowe. On remarque que, dans la représentation logarithmique adoptée, les droites de régression sont caractérisées par un coefficient de corrélation supérieur à 0,97 . Cela permet, dans le cas des essais avec.un palier de chargement cyclique, d'évaluer la valeur de $\mathrm{t}_{50}$ que l'on aurait obtenue sous un chargement statique correspondant au même palier à partir des valeurs $t_{50}$ mesurées lors des paliers précédents et suivants. Les résultats obtenus sur la cellule de Rowe indiquent des vitesses de consolidation supérieures à celles mesurées à l'œdomètre.

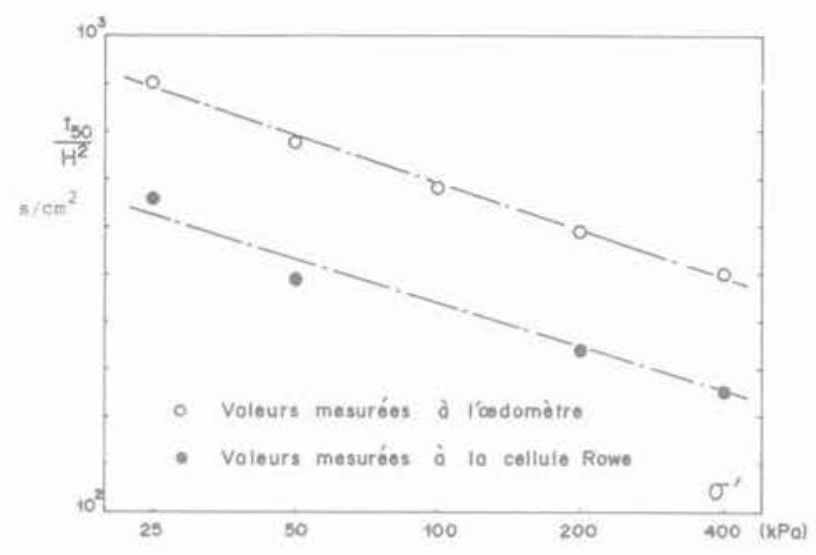

Fig. 8 - Variations des valeurs moyennes de $t_{50} / H^{2}$ avec la contrainte effective.

Certains essais de consolidation ont été effectués à la cellule perméamétrique en mesurant, à la fin de chaque palier, la perméabilité du sol par un essai à charge variable. Pour ces essais, on peut calculer à chaque palier, la valeur du rapport $t_{50} / \mathrm{H}^{2}$ à partir de la théorie de TERZAGHI en considérant les valeurs expérimentales des caractéristiques de compressibilité $\mathrm{a}_{y}$ et de perméabilité $k$ du sol. D'après la théorie de TERZAGHI ce rapport est donné par :

$$
\frac{\mathrm{t}_{50}}{\mathrm{H}^{2}}=\mathrm{T}(50 \%) \frac{\mathrm{a}_{v} \gamma_{w}}{\mathrm{k}\left(1+\mathrm{e}_{\mathrm{o}}\right)}
$$

En comparant, pour différents paliers de charge, la valeur mesurée du rapport $t_{50} / \mathrm{H}^{2}$ à celle calculée on remarque que la théorie conduit à sous-estimer les temps de consolidation.

La figure 9 montre une comparaison entre les variations de l'indice des vides en fonction de la contrainte effective mesurée à l'œedomètre et à la cellule de Rowe. Les 
écarts observés pour les paliers de faibles contraintes $(<20 \mathrm{kPa})$ sont essentiellement dus à l'imprécision des mesures ayant pour origine des difficultés de réglage du système de mise en pression à la cellule de Rowe sous faibles charges, des frottements parasites, etc.

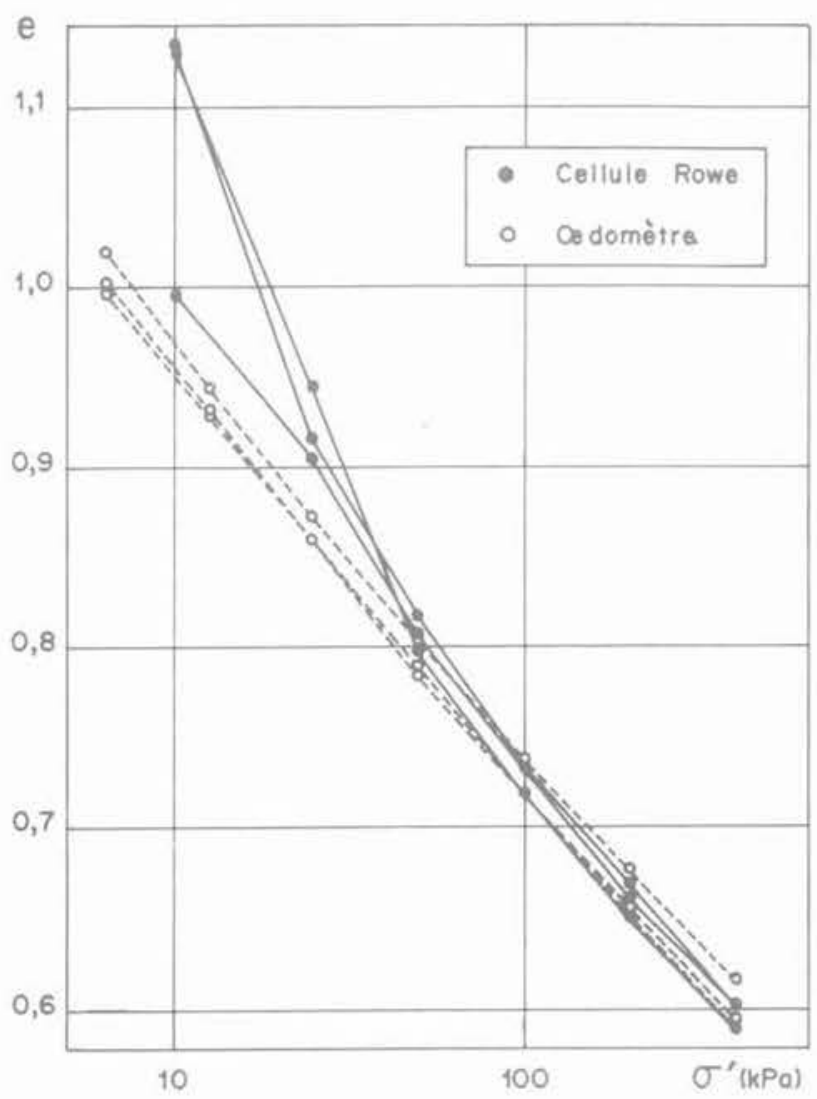

Fig. 9 - Courbes œdométriques.

Il est à noter que le tassement final $\Delta \mathrm{h}_{f}$ décroît linéairement avec Log $\sigma_{f}^{\prime}\left(\sigma^{\prime}\right.$, étant la contrainte effective en fin de palier). L'analyse de l'ensemble des essais à l'cedomètre a montré que la droite de régression de cette variation linéaire présente un coefficient de corrélation supérieur à 0,99. Cela permet lors des essais avec un palier de chargement cyclique d'évaluer le tassement qui aurait été provoqué par un chargement statique correspondant au même palier à partir des valeurs de tassements mesurées lors des paliers précédents.

\subsection{Détermination expérimentale des paramètres $\alpha$ et $\beta$}

D'après la théorie de BALIGH et LEVADOUX, le comportement réel d'un sol peut être schématisé par une relation bilinéaire entre l'indice des vides et la contrainte effective, en considérant respectivement le comportement du sol à l'état normalement consolidé et à l'état surconsolidé. Ce comportement est alors caractérisé par les deux paramètres :

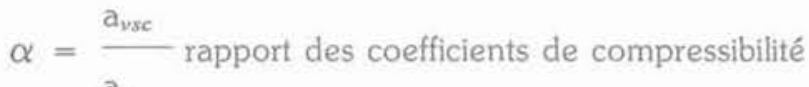

$\beta=\frac{C_{v}}{C_{v s c}}$ rapport des coefficients de consolidation.

Pour déterminer les valeurs expérimentales de ces paramètres on a effectué à la cellule perméamétrique des essais œedométriques au cours desquels chaque palier de contrainte a comporté trois phases de 24 heures chacune :

- Phase 1: premier chargement sous une contrainte $\sigma_{f}^{\prime}=\sigma_{p}^{\prime}+\Delta \sigma$

$\sigma_{p}$ étant la contrainte de préconsolidation du palier,

- Phase 2: déchargement et retour à la contrainte $\sigma_{p}^{\prime}$ du palier,

- Phase 3: second chargement sous la contrainte $\sigma_{f}^{\prime}$ avant de passer au palier suivant.

A l'issue de chacune de ces phases, on a mesuré la perméabilité du sol par un essai à charge variable.

Les résultats de ces essais sont présentés sur la figure 10. Ils montrent que la perméabilité et la compressibilité varient en fonction de la contrainte effective. On remarque également que les paramètres $\alpha$ et $\beta$ définis par BALIGH et LEVADOUX ne sont pas constants.
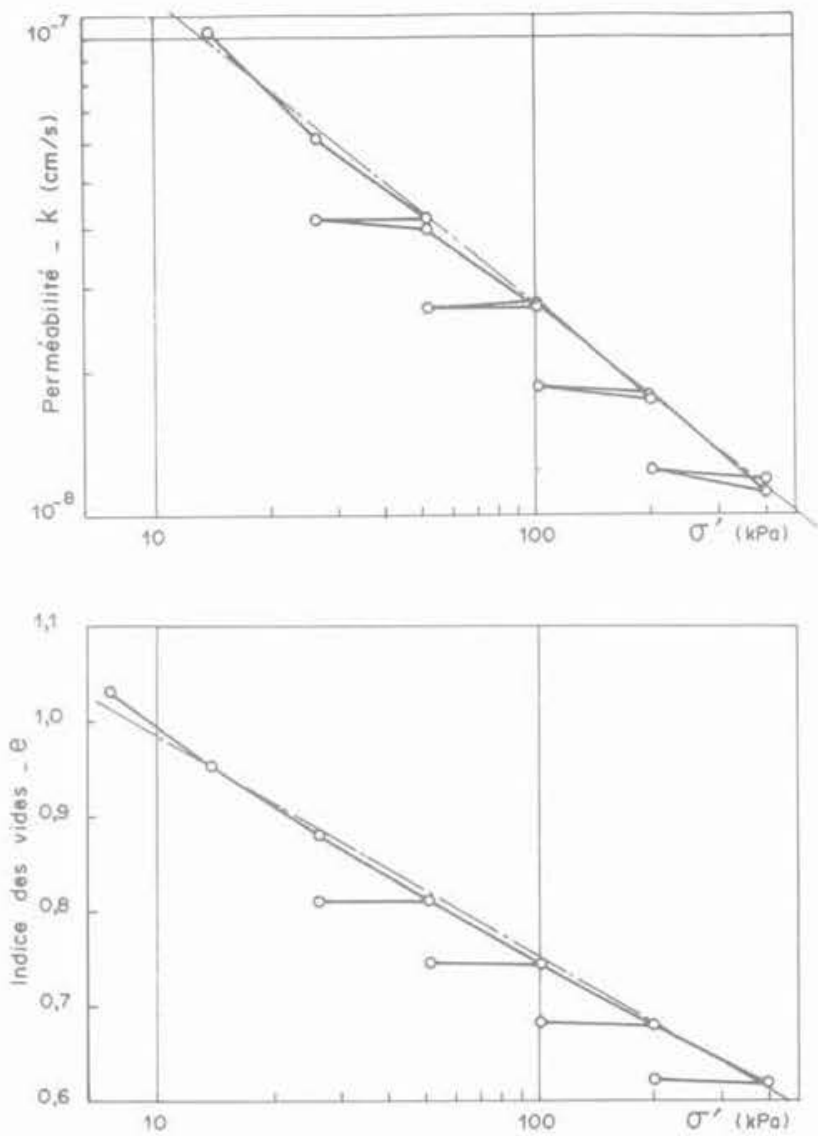

Fig. 10 - Variations de la perméabilité et de l'indice des vides en fonction de la contrainte effective $\sigma^{\prime}$.

S’il est aisé de déterminer la variation du paramètre $\alpha$ avec la contrainte effective, la détermination expérimentale directe du paramètre $\beta$ s'avère plus difficile car la 
consolidation du sol a l'état surconsolidé est très rapide et ne permet pas une évaluation précise du coefficient $\mathrm{C}_{v s e}$ à partir des mesures de tassement. On peut cependant évaluer ce coefficient à partir des mesures de la perméabilité. On remarque que :

$\beta_{\min }=\alpha<\beta<\beta_{\max }=\alpha \frac{\mathrm{k}\left(\sigma_{p}^{\prime}\right)}{\mathrm{k}\left(\sigma_{f}^{\prime}\right)}$

où : $\sigma_{p}^{\prime}=$ contrainte de préconsolidation du palier, $\sigma_{f}^{\prime}=$ contrainte effective à la fin du palier.

Les variations des paramètres $\alpha$ et $\beta$ en fonction de la contrainte effective sont présentées sur la figure 11.

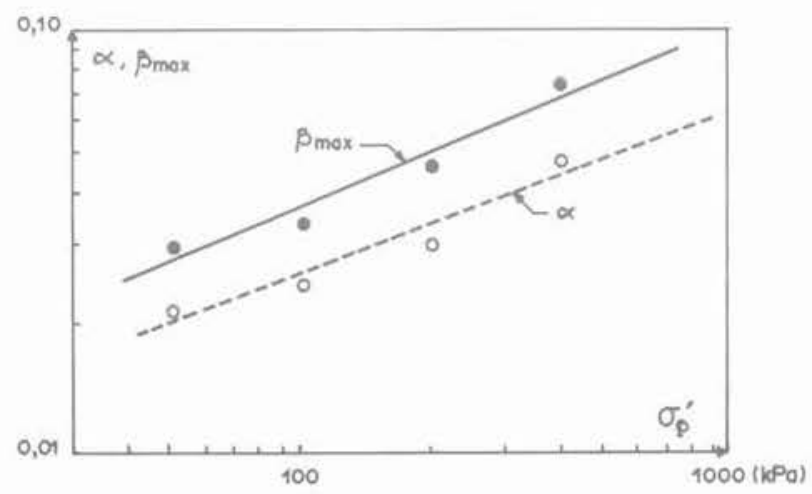

Fig. 11 - Variation de $\alpha$ et $\beta$ en fonction de $\sigma_{p}^{\prime}$.

Dans le cas de la cellule de Rowe, il est aussi simple de déterminer un temps caractéristique $\left(_{50}\right.$ en l'occurrence) sur les courbes de dissipation de la pression interstitielle pour le sol à l'état surconsolidé que pour le sol à l'état normalement consolidé. Pour une majorité des essais effectués entre 200 et $400 \mathrm{kPa}$, les valeurs obtenues pour $\beta$ sont comprises entre 0,05 et 0,09 environ et sont donc du même ordre de grandeur que celles présentées sur la figure 11.

\subsection{Résultats des essais sous charges cycliques}

\subsubsection{Présentation}

Les figures 12 et 13 montrent des courbes de consolidation obtenues aussi bien sur un œdomètre classique que sur la cellule de Rowe lors d'essais sous charge cyclique. Ces courbes sont présentées sous la forme d'un degré de consolidation $U$ en fonction d'un facteur temps $\mathrm{T}$,

où : $\mathrm{U}=\frac{\Delta \mathrm{h}}{\Delta \mathrm{h}_{\mathrm{c}}}$

La valeur de $\Delta h_{c}$ prise en compte est celle du tassement final obtenu à la fin de la consolidation primaire sous la charge maximale maintenue constante après Parrêt des cycles.

et : $\mathrm{T}=\mathrm{T}(50 \%) \cdot \mathrm{t} / \mathrm{t}_{50}$ avec $(\mathrm{T}(\mathrm{t} 50 \%)=0.197)$. où : $t_{50}$ est la valeur estimée pour le temps de consolidation sous charge statique à partir des valeurs de $t_{50}$ mesurées lors des paliers précédents et suivants.

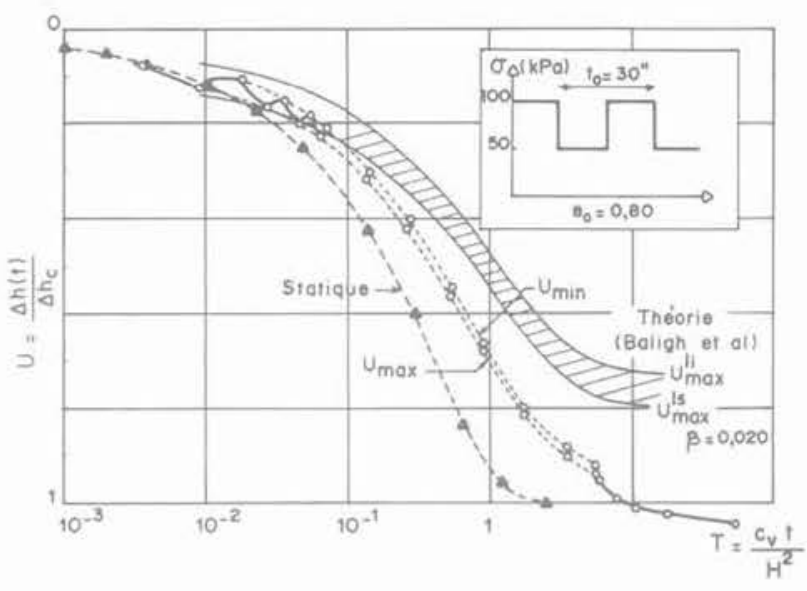

Fig. 12 - Courbes de consolidation sous charges statique et cyclique à l'cedomètre.

Comparaison théorie-expérience.

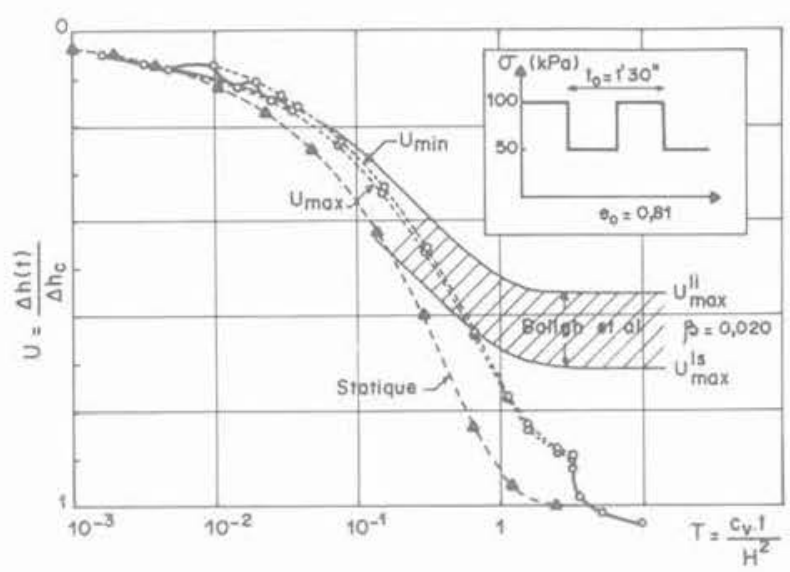

Fig. 13 - Courbes de consolidation sous charges statique et cyclique à la cellule Rowe.

Comparaison théorie-expérience.

On remarque que lallure générale de ces courbes est très proche de celle prévue par la solution de BALIGH et LEVADOUX en considérant les valeurs expérimentales des paramètres $\alpha$ et $\beta$. Le tassement oscille à l'intérieur d'un fuseau, qui s'est révélé très étroit, défini par deux courbes enveloppes. L'allure de ces courbes montre que, d'une façon générale, la charge cyclique a été maintenue suffisamment longtemps pour que le phénomène de consolidation primaire soit arrivé à son terme et que les tassements restant à intervenir ne correspondent qu'au phénomène de consolidation secondaire. autrement dit on a atteint un état d'équilibre à pression interstitielle moyenne constante. Ce point est confirmé par les mesures des surpressions interstitielles à la cellule de Rowe. A larrêt des cycles on maintient la contrainte maximale $(100 \mathrm{kPa})$ constante. On observe alors une reprise du tassement de consolidation primaire due à une dissipation des surpressions interstitielles. Ces résultats sont en accord avec les résultats théoriques indiquant qu'à l'état d'équilibre sous charge cyclique, la couche de sol n'est pas entièrement consolidée $\left(\mathrm{U}^{\infty}{ }_{\max }<1\right)$. 


\subsubsection{Etude paramètrique}

La variable principale de cette étude est la période des cycles $t_{o}$ exprimée en facteur temps pour le sol à l'état normalement consolidé, soit : $\mathrm{T}_{O}=\mathrm{C}_{v} \cdot \mathrm{t}_{o} / \mathrm{H}^{2}$. Les essais sous charges cycliques avec des périodes relativement longues $\left(\mathrm{T}_{0}>10^{-2}\right)$ ont été effectués à l'œedomètre classique. Pour effectuer des cycles de courte durée on a utilisé la cellule de Rowe. On présente ciaprès les résultats expérimentaux concernant l'effet des cycles sur le degré de consolidation à l'état d'équilibre sous la charge cyclique, sur le tassement final, sur la vitesse de consolidation et sur la dissipation des surpressions interstitielles.

\section{DEGRE DE CONSOLIDATION}

\section{A L'ETAT D'EQUILIBRE SOUS CHARGE CYCLIQUE}

La théorie de BALIGH et LEVADOUX montre que les degrés de consolidation $U^{\infty}{ }_{\max }$ et $U^{\infty}{ }_{\min }$ atteints à l'état d'équilibre sous charge cyclique sont fonction de la période des cycles.

La figure 14 montre les valeurs expérimentales de ces degrés de consolidation, mesurées à l'arrêt des cycles, en fonction de la période $T_{o}$. Les valeurs expérimentales sont comparées aux courbes théoriques calculées en utilisant les valeurs de $\alpha$ et $\beta$ déterminées à partir d'essais sous charge statique $\beta=0,020$ et $\alpha=0,025$. Cette comparaison montre que, comme le prévoit la théorie, la valeur de $U^{\infty}{ }_{\max }$ croît avec la période. Cependant, les valeurs théoriques de $U^{\infty}{ }_{\max }$ obtenues en utilisant la valeur expérimentale du paramètre $\beta$ sont nettement inférieures à celles observées expérimentalement.

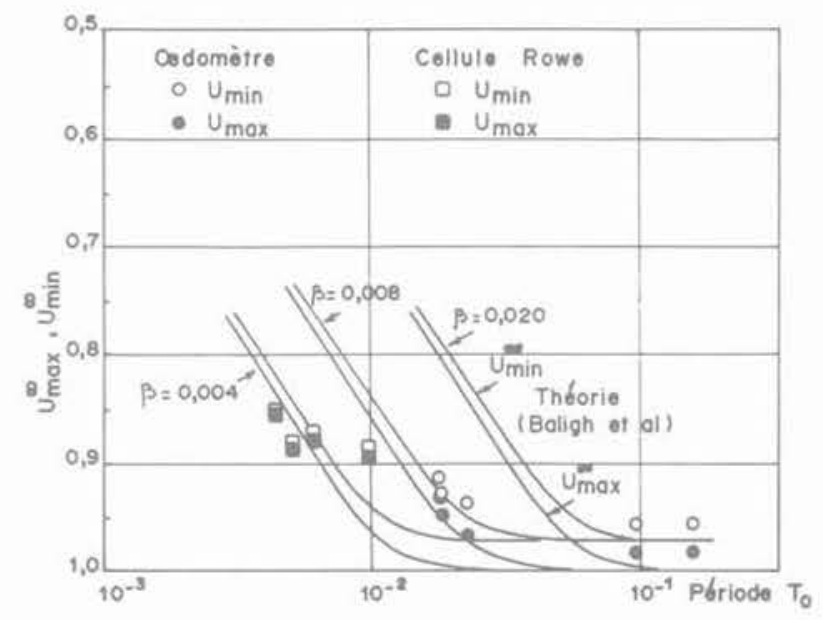

Fig. 14 - Degrés de consolidation maximal et minimal à l'état d'équilibre.

Il est possible de déterminer pour chaque essai une valeur de $\alpha$ à partir de l'équation (4). Les valeurs de $\alpha$ ainsi déterminées à partir de l'ensemble des essais cycliques sont regroupées sur la figure 15 . On remarque que, si les valeurs déduites des essais à l'œdomètre classique correspondent à la valeur déterminée à partir des essais sous charge statique (fig. 11), celle-ci est nettement supérieure aux valeurs obtenues dans les essais à la cellule de Rowe. Cela traduit dans le cas de la cel- lule de Rowe un regonflement plus faible que ne le prévoit la théorie; cet écart peut éventuellement être attribué à l'existence de frottements parasites.

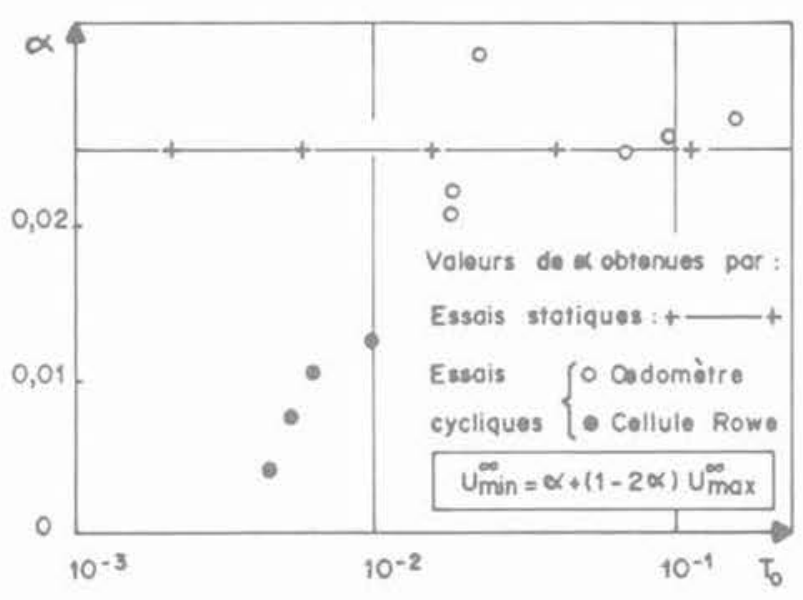

Fig. 15 - Comparaison des valeurs du paramètre $\alpha$ déduites d'essais sous charges statique et cyclique.

\section{TASSEMENT FINAL $-\Delta h_{c}$}

La figure 16 montre l'effet des cycles sur le tassement total $\Delta \mathrm{h}_{\mathrm{c}}$ obtenu après arrêt des cycles. On remarque que le rapport $\Delta h_{c} / \Delta h_{f}\left(\Delta h_{f}\right.$ étant le tassement final correspondant à un essai sous charge statique) croît légèrement avec la période des cycles $T_{0}$, mais l'effet sur l'amplitude des tassements reste inférieur à $10 \%$.

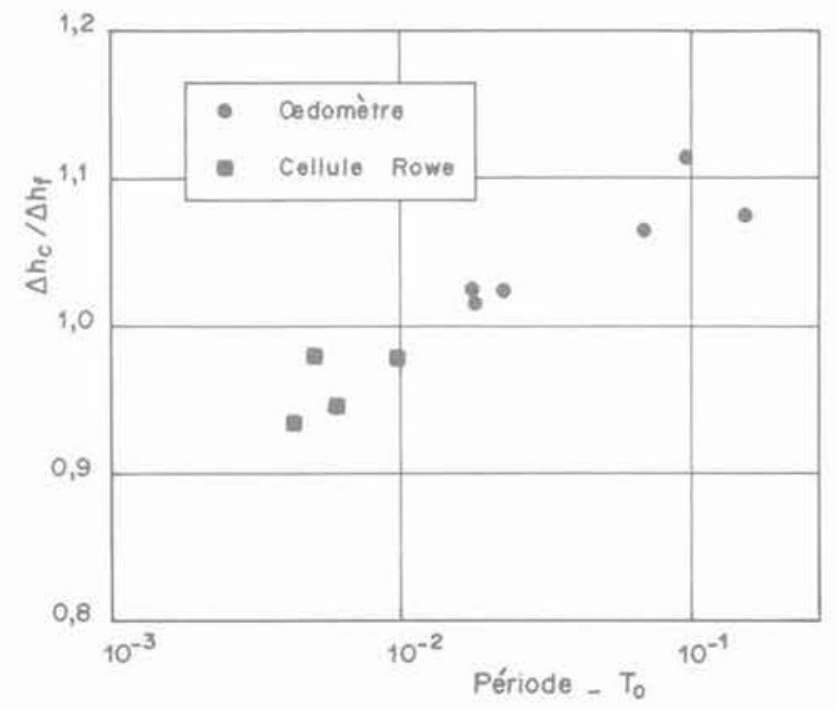

Fig. 16 - Effet des cycles sur le tassement total après l'arrêt des cycles.

\section{VITESSE DE CONSOLIDATION}

Sur les figures 12 et 13 , on compare les courbes de consolidation sous charge cyclique avec celles obtenues sous la contrainte statique maximale. On remarque que, comme le prévoit la théorie, les cycles ont pour effet de 
ralentir la consolidation. Pour caractériser cet effet des cycles, on a déterminé sur les courbes de consolidation cyclique le temps $t_{50 c}$ nécessaire pour atteindre un degré de consolidation de $50 \%$ défini par rapport au tassement total après arrêt des cycles $\Delta h_{c}$. Les valeurs du rapport $t_{50 c} / t_{50 s}$ (où $t_{50 s}$ correspond en un essai sous charge statique) sont regroupées sur la figure 17 en fonction de la période des cycles $T_{O}$. On remarque que, pour l'ensemble des essais à l'øedomètre et à la cellule de Rowe la valeur de ce rapport se situe aux environs de 2. On observe ainsi pratiquement un doublement des temps de consolidation sous cycles par rapport à la consolidation sous charge statique.

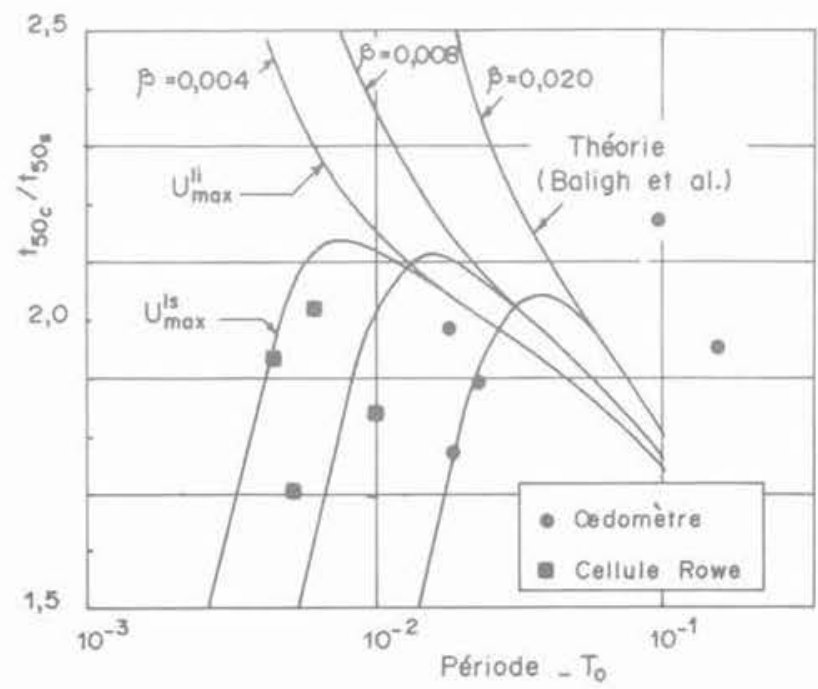

Fig. 17 - Effet des cycles sur la vitesse de consolidation.

La comparaison entre les courbes théoriques de consolidation sous charge cyclique et les courbes expérimentales présentées sur les figures 12 et 13 montre que la théorie de BALIGH et LEVADOUX conduit à sous-estimer sensiblement la vitesse de consolidation et le degré de consolidation à l'état d'équilibre. Il est à noter que, pour la période des cycles considérée $\left(T_{0}=10^{-2}\right)$, l'écart entre les bornes inférieure et supé. rieure, calculées en utilisant la valeur expérimentale de $\beta(\beta=0,020)$, devient important.

Sur la figure 17, on compare les valeurs expérimentales du rapport $t_{50 c} / t_{50 s}$ avec les valeurs théoriques calculées pour différentes valeurs du paramètre $\beta$. L'écart entre les bornes inférieure $\left(U_{\text {max }}^{\mathrm{l}}\right)$ et supérieure $\left(U^{l s}{ }_{\max }\right)$ devient important pour les périodes courtes et rend alors toute comparaison difficilement interprétable.

\section{PRESSION INTERSTITIELLE SOUS CHARGE CYCLIQUE}

La figure 18 montre pour un essai de consolidation sous charge cyclique la variation de la contrainte effective à la base de l'échantillon déduite des mesures de pressions interstitielles à la cellule de Rowe. On peut distinguer trois phases :

- une phase transitoire qui aboutit sur un état d'équilibre :

- un état d'équilibre caractérisé par des variations de la pression identiques d'un cycle à l'autre :
- une courbe de dissipation après arrêt des cycles lorsque la charge maximale appliquée $(100 \mathrm{kPa})$ est maintenue constante.

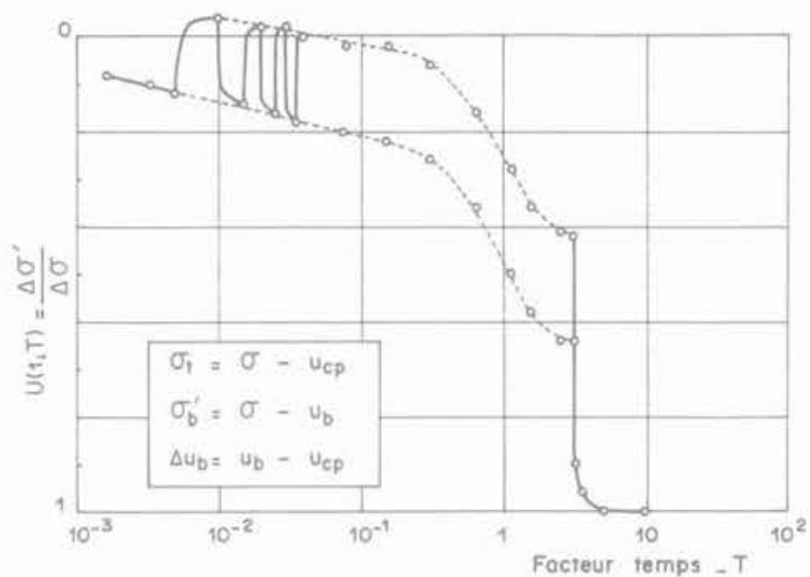

Fig. 18 - Variation de la contrainte effective mesurée lors d'une consolidation sous charge cyclique.

Pendant la phase transitoire, la contrainte effective mesurée à la base de l'échantillon croit lorsque le sol est chargé et décroît lorsqu'il est déchargé. Elle oscille donc à l'intérieur d'un fuseau défini par deux courbes enveloppes tendant chacune vers une valeur limite lorsqu'on atteint l'état d'équilibre. L'écart entre les deux reste pratiquement constant tout au long de la consolidation.

Sur la figure $19 \mathrm{a}$, on compare la variation expérimentale de la contrainte effective à la base de l'éprouvette au cours d'un cycle à l'état d'équilibre avec celle déduite de la théorie de TERZAGHI en tenant compte des observations formulées par BALIGH et LEVADOUX.

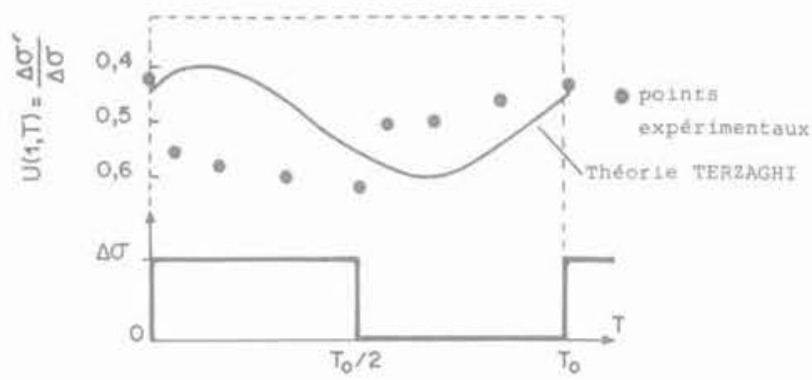

Fig. 19a - Variation de la contrainte effective à la base à l'état d'équilibre.

D'après la théorie, lorsqu'on a atteint un état d'équilibre, la totalité de la couche de sol a un comportement suconsolidé et la répartition des contraintes et des pressions interstitielles est alors identique à celle obtenue à partir de l'équation (1) en considérant un sol à comportement réversible avec une période de cycle $T_{0} / \beta$.

La comparaison entre la théorie et l'expérience fait apparaître certaines différences entre les variations calculées et mesurées de la contrainte effective. Cependant, les valeurs théoriques des contraintes effectives maximale et minimale atteintes au cours des phases de chargement 
et de déchargement correspondant aux valeurs mesurées. On peut en particulier noter que, comme le suppose la théorie (équation (2)), on a pratiquement : $\Delta \sigma_{\max }^{\prime}+\Delta \sigma_{\min }^{\prime}=\Delta \sigma$.

Par ailleurs, d'après la théorie. l'amplitude de la variation de la pression intersticielle n'est fonction que de la période des cycles $T_{o} / \beta$. La figure $19 \mathrm{~b}$ montre que les valeurs expérimentales de cette amplitude correspondent aux prévisions théoriques obtenues en prenant pour $\beta$ sa valeur expérimentale $\beta=0,020$ (fig. 11).

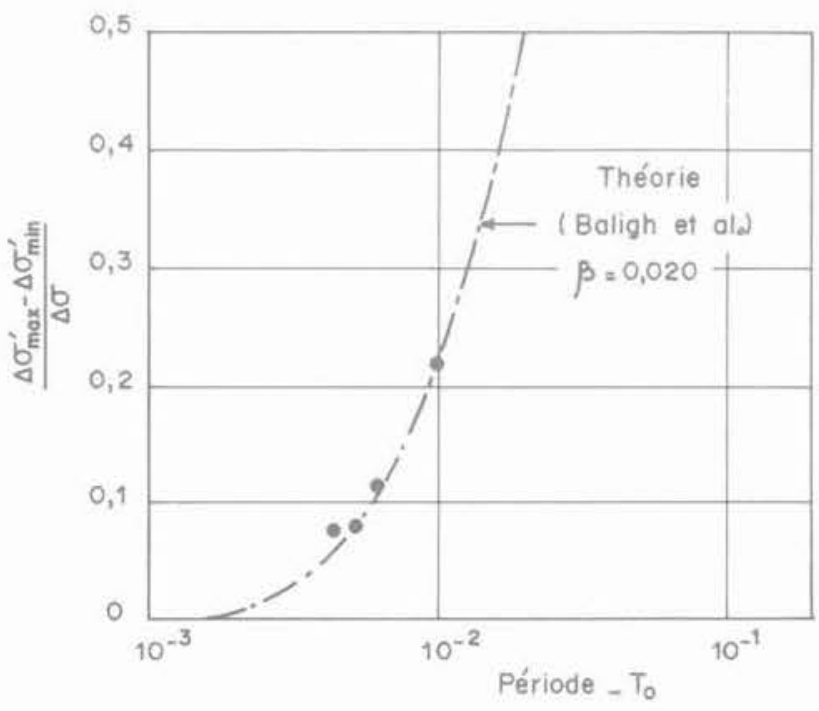

Fig. $19 b$ - Amplitude de la variation de la contrainte effective à la base à l'état d'équilibre.
Enfin, il est intéressant de noter que la comparaison entre la théorie et l'expérience fait apparaitre un bon accord entre les valeurs mesurées et calculées des pressions interstitielles à l'état d'équilibre, alors que la théorie conduit à sous-estimer le tassement à l'état d'équilibre et la vitesse de consolidation. Ces différences peuvent être attribuées, du moins partiellement, à l'effet sur le tassement de l'irréversibilité du comportement du sol à l'état surconsolidé sous charge cyclique.

En effet, cette irréversibilité a été mise en évidence par un essai à l'cedomètre classique ou un chargement statique $\left(\sigma_{p}^{\prime}=200 \mathrm{kPa} ; \Delta \sigma=200 \mathrm{kPa}\right)$ a été suivi d'une succession de déchargements et de chargements (entre 100 et $400 \mathrm{kPa}$ ) dont la période (durée de chargement $\sim 100 \mathrm{~min}$., durée de déchargement $\sim 4$ min.) était suffisante pour une dissipation complète des surpressions interstitielles. La figure 20 a montre la courbe de consolidation correspondante. La figure $20 \mathrm{~b}$ montre les courbes de consolidation obtenues lors des différentes phases de chargement, le sol étant entièrement surconsolidé. On remarque l'apparition de tassements irréversibles liés probablement au phénomène de fluage du sol (consolidation secondaire). En effet, lors d'un rechargement la consolidation primaire est pratiquement terminée avant d'atteindre le tassement obtenu lors de la phase précédente. Comme le montre la figure 20a l'accumulation de ces tassements irréversibles du sol à l'état surconsolidé, s'effectue à une vitesse supérieure à celle de la consolidation secondaire du sol normalement consolidé.

Pour le chemin de contrainte unidimensionnel suivi lors d'un essai œdomètrique, ce comportement irréversible à l'état surconsolidé ne semble pas avoir un effet sensible sur les pressions interstitielles, en particulier, à l'état d'équilibre ; il a, par contre, une influence significative sur les tassements totaux. La modélisation adoptée par BALIGH et LEVADOUX ne permet pas de tenir compte de l'irréversibilité du comportement du sol à l'état surconsolidé et conduit donc à sous-estimer les tassements.

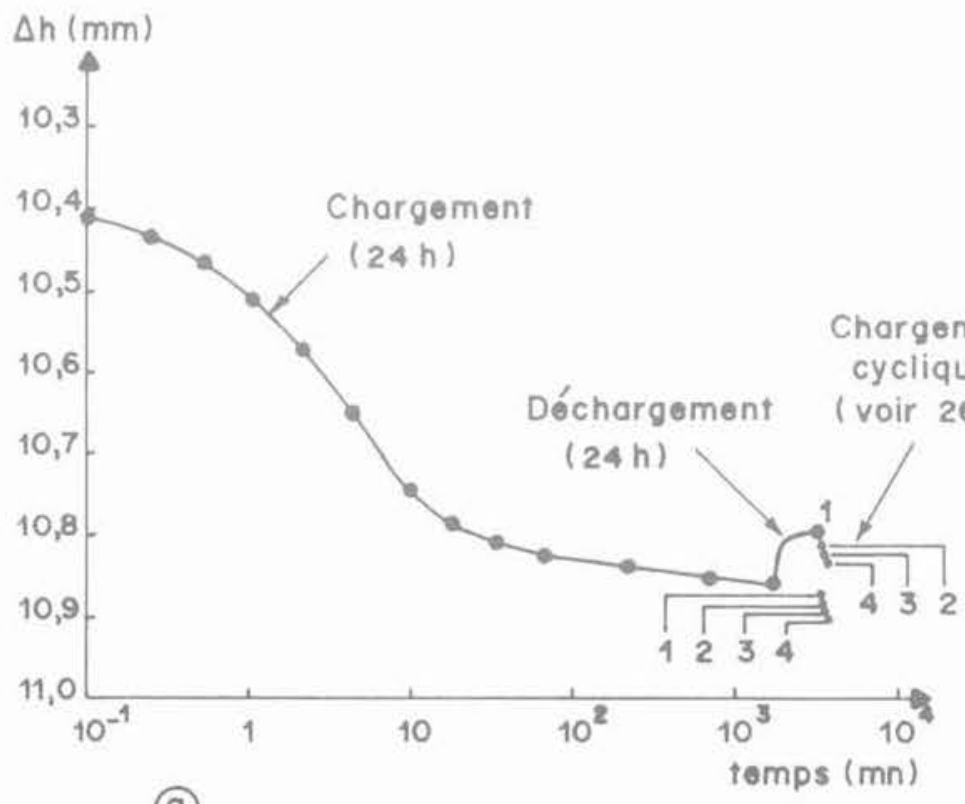

(a)

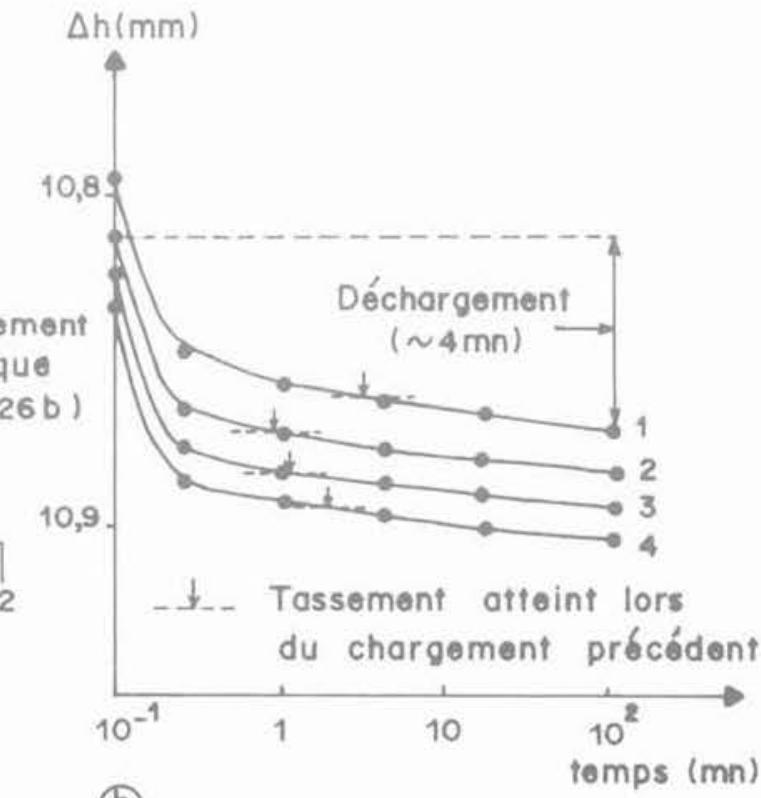

(b)

Fig. 20 - Résultats d'un essai cyclique de longue période à l'œedomètre. 


\section{CONCLUSIONS}

Cette étude expérimentale a permis de mettre en évidence un certain nombre des phénomènes intervenant dans la consolidation unidimensionnelle sous charge cyclique. On remarque en particulier que les cycles ont pour effet de réduire la vitesse de consolidation. Lors d'une consolidation sous un chargement cyclique le sol atteint un état d'équilibre, à pression interstitielle moyenne constante, sans que les surpressions interstitielles provoquées par le chargement appliqué soient entièrement dissipées. Ainsi, à l'état d'équilibre sous la charge cyclique appliquée le sol n'est pas entièrement consolidé. En maintenant, à l'arrêt des cycles, la contrainte maximale appliquée constante, on provoque une reprise du tassement de consolidation primaire due à une dissipation de la surpression interstitielle qui réside dans l'éprouvette à l'état d'équilibre sous la charge cyclique. La comparaison des résultats expérimentaux avec la solution théorique de BALIGH et LEVADOUX montre que si cette solution permet une interprétation qualitative des phénomènes observés, elle conduit à sousestimer considérablement la vitesse de consolidation et le degré de consolidation à l'état d'équilibre sous la charge cyclique. En effet, cette solution est fondée sur les hypothèses restrictives de la théorie de TERZAGHI et ne permet pas de rendre compte fidèlement du comportement irréversible du sol lors des cycles de chargement et de déchargement. Par ailleurs, cette solution n'est pas adaptée à l'étude de la consolidation sous cycles de courtes périodes.

Enfin, il est à noter que cette étude n'a concerné que l'effet de la période des cycles et sa poursuite est nécessaire pour mieux comprendre l'effet d'autres paramètres de chargement (amplitude des cycles, palier de contraintes, etc.) et en particulier de la nature du sol.

\section{REMERCIEMENTS}

Cette recherche est financée par la D.A.E.I. dans le cadre d'un projet commun à I'E.N.P.C. et à PUniversité du Colorado. Il a été mené en liaison étroite avec des recherches technologiques effectuées par la Société "Techniques Louis Ménard " afin de mettre au point une nouvelle technique de consolidation par un chargement cyclique radial associé à des drains verticaux. Les auteurs remercient, en particulier, MM. COGNON et LIAUSU de la société Solcompact pour leurs conseils au cours de cette étude, le Professeur SCHIFFMAN de I'Université du Colorado pour de nombreuses discussions sur l'interprétation des essais effectués, et $M$. J.P. MAGNAN du Laboratoire Central des Ponts et Chaussées pour une lecture critique et constructive du projet de cet article.

\section{BIBLIOGRAPHIE}

1. BALIGH M.-M., LEVADOUX J.-N., (1978), Consolidation theory for cyclic loading. Journal of the geotechnical engineering division, ASCE, Vol. 104, n GT4, April 1978, pp. 415-431.

2. WILSON N.-E., ELGOHARY M.-M., (1974), Consolidation of soils under cyclic loading. Canadian Geotechnical Journal, 11, pp. 420-423.

3. ROWE P.-W., BARDEN L., (1966), A new consolidation cell. Géotechnique, Vol. 16, $\mathrm{n}^{\circ} 2$, juin 1966, pp. 162-170.

4. DEERE D.-U., DAVISSON M.-T., (1961), Behavior of grain elevator foundations subjected to cyclic loading. Proc. 5th I.C.S.M.F.E. Paris, Vol. 1, pp. $629 \cdot 633$.

5. JAMBU N., SENNESET K., (1981), Settlements due to drained cyclic loads. Proc. Xe I.C.S.M.F.S., Stockholm.

6. TAVENAS F., BRUCY M., MAGNAN J.-P., LA ROCHELLE P., ROY M., (1979), Analyse critique de la théorie de consolidation unidimensionnelle de TERZAGHI. Revue Française de Géotechnique n० 7, pp. 29-43, -

7. WILSON N.-E., GREENWOOD J.-R., (1974), Pore pressures and strains after repeated loading of saturated clay. Canadian Geotechnical Journal, 11. pp. 269-277.

8. SCHLOSSER F., (1973), Hypothèses et théories pour la prévision des tassements des remblais sur sols compressibles, Bull. de Liaison des Laboratoires des Ponts et Chaussées, Spécial T. Remblais sur sols compressibles, L.C.P.C., Paris. 